\title{
Sex Difference in Adaptation of Serum Calcium Level to the Gradual Deprivation of Parathyroid Hormone and to the Following Thyroidectomy
}

\author{
Tомоко FUJII AND KUMIKo MORITA \\ Department of Pharmacology, Teikyo University \\ School of Medicine, Tokyo 173
}

\begin{abstract}
The tıme course of adaptation of the serum calcium level to the gradual deprivation of parathyroid hormone was studied in adult male and female rats. In addition, the role of thyrocalcitonin and thyroid hormones in adaptive changes in serum calcium was assessed in the surgically parathyroidectomized animals. Simultaneous bilateral parathyroidectomy, thyroparathyroidectomy or the second unilateral parathyroidectomy performed $72 \mathrm{hr}$ after the first unilateral parathyroidectomy produced a prompt fall in serum calcium level leading to the lowest value at $10 \mathrm{hr}$ and the significantly lower calcium level retained for one week-observation. Unilateral parathyroidectomy resulted in a transient fall in the serum calcium level and to a rapid return to the subnormal range in $24 \mathrm{hr}$ in both sexes. When the second unilateral parathyroidectomy was carried out 1 or 6 weeks after the first unilateral parathyroidectomy, the serum calcium in female rats showed a slight decrease until $10 \mathrm{hr}$ and returned to the normal level in one week, but in male rats no further decline or a gradual decrease was seen. Bilateral thyroidectomy performed 10 weeks after the second unilateral parathyroidectomy resulted in a gradual increase in the serum calcium above the normal range in female rats. In male rats, the serum calcium level tended to drop until $10 \mathrm{hr}$ and returned to the initial level or to the normal range. Bilateral thyroidectomy performed one week after simultaneous bilateral parathyroidectomy led to a further decline in serum calcium in both male and female rats. The results indicate that adaptation of the serum calcium level toward the normal range occurs in the absence of parathyroid hormone in adult male and female rats if the parathyroid hormone is gradually deprived. A greater ability of female rats to restore the calcium level, especially seen when thyroidectomized after adaptation to the lack of parathyroid hormone, suggests that estrogens play a significant role in the homeostatic control of serum calcium in chronic disorders in calcium regulation.
\end{abstract}

Adult female rats born to parathyroidectomized mothers show a less marked fall in the serum calcium level following unilateral or bilateral parathyroidectomy as compared with that seen in intact adult females (Fujii, 1978). This finding suggests that the functional differentiation of organs which regulate calcium metabolism is affected by the uterine environment supplied with the blood of low calcium.

Received March 9, 1979.
It is well established that parathyroid hormone, calcitonin and metabolites of vitamin $\mathrm{D}_{3}$ play a major role in the homeostatic control of serum calcium. Bone, kidney and intestine have been considered to be responsible for the regulation of serum calcim level (for Review see Copp, 1974). However, precise interrelationship among organs involved in calcium homeostasis is still unclear.

The present experiment was carried out to study the time course of the adaptation 
of the regulatory system of serum calcium to the lack of parathyroid hormone in intact adult rats. A gradual decrease in the serum calcium level was induced by stepwise removal of parathyroid gland with various time intervals. Serum calcium level was used as a parameter of alteration of calcium homeostasis. In addition, the effect of thyroidectomy was also studied in order to know the contribution of both thyrocalcitonin (calcitonin) and thyroid hormones in adaptation of calcium metabolism to the lack of parathyroid hormones.

\section{Materials and Methods}

Adult male rats, weighing $300-350 \mathrm{~g}$, and female rats, weighing $230-260 \mathrm{~g}$, of the inbred Wistar-Imamichi strain, were obtained from the Animal Breeding Laboratory (Ohmiya, Saitama Prefecture) and kept in our laboratory at least one week before use. In the first experiment, male and female rats were subjected to unilateral parathyroidectomy and then the rest of their parathyroid gland was removed $72 \mathrm{hr}$, one or six weeks later. Bilateral thyroid glands in rats of these three groups were removed 10 weeks after the second unilateral parathyroidectomy.

In the second experiment, rats of both sexes were divided into two groups; one group received simultaneous bilateral parathyroidectomy and was followed by bilateral thyroidectomy one week later; another group received simultaneous bilateral thyroparathyroidectomy. Control animals were sham-operated twice with a time interval of one week. The surgical parathyroidectomy or thyroidectomy in all experimental groups were performed under ether anesthesia, starting around 0930 a.m. for all experimental groups. All animals were given stock diet (Calcium, $1.01 \%$; phosphorus, $1.17 \%$, Oriental Yeast Co. Ltd., Tokyo) and tap water ad libitum and kept in a temperature-controlled room $\left(21-25^{\circ} \mathrm{C}\right)$ with a lighting schedule of $14 \mathrm{hr}$ light (0600 a.m.-0800 p.m.) and $10 \mathrm{hr}$ darkness. A branch of the saphenous vein of the hind-limb was punctured by a 22-gauge needle and blood samples were collected 2, 6, 10, 24, 48 and $72 \mathrm{hr}$ and 7 days after each operation without anesthesia. The sera isolated were stored at $-20^{\circ} \mathrm{C}$ until analyzed. Calcium concentration was determined using a Hitachi atomic absorption spectrometer. Serum protein was measured by the method of Lowry et al. (1951). The statistical analyses employed were Studen's $t$ test ; $\mathrm{P}<0.05$ was considered to be significant.

\section{Results}

Effects of stepwise removal of parathyroid gland and the following bilateral thyroidectomy

The serum calcium level decreased following the first unilateral parathyroidectomy in male and female rats, reaching the lowest level at $10 \mathrm{hr}$ and it returned toward the subnormal level at $24 \mathrm{hr}$ (Table 1), and no sex difference was shown.

When the second unilateral parathyroidectomy was carried out $27 \mathrm{hr}$ after the first unilateral parathyroidectomy, serum calcium level showed a rapid and prolonged decline, reaching the lowest level at $10 \mathrm{hr}$. The magnitude of the decline in female rats was greater than that in males until $24 \mathrm{hr}$. But the calcium level in male rats

Table 1. Time course changes in serum calcium levels $(\mathrm{mg} / 100 \mathrm{ml})$ following unilateral parathyroidectomy and bilateral thyroparathyroidectomy in adult rats

\begin{tabular}{ccccc}
\hline $\begin{array}{c}\text { Time after } \\
\text { operation }\end{array}$ & \multicolumn{2}{c}{$\begin{array}{c}\text { Unilateral parathyroidectomy } \\
\text { Male }(8)^{\mathrm{a}}\end{array}$} & $\begin{array}{c}\text { Bilateral thyroparathyroidectomy } \\
\text { Female }(8)\end{array}$ & $\begin{array}{c}\text { Male (4) } \\
\text { Female }\end{array}$ \\
\hline $0 \mathrm{hr}$ & $10.92 \pm 0.24^{\mathrm{b}}$ & $10.90 \pm 0.21$ & $10.99 \pm 0.21$ & $11.35 \pm 0.20$ \\
2 & $10.35 \pm 0.13$ & $10.92 \pm 0.12$ & $10.56 \pm 0.31$ & $11.32 \pm 0.16$ \\
6 & $9.60 \pm 0.42^{*}$ & $9.25 \pm 0.08^{* *}$ & $8.86 \pm 0.38^{* *}$ & $9.50 \pm 0.42^{* *}$ \\
10 & $8.97 \pm 0.28^{* *}$ & $8.76 \pm 0.14^{* *}$ & $8.05 \pm 0.61^{* *}$ & $8.99 \pm 0.63^{*}$ \\
24 & $9.35 \pm 0.12^{* *}$ & $9.38 \pm 0.16^{* *}$ & $9.38 \pm 0.62^{*}$ & $9.61 \pm 0.63$ \\
48 & $9.55 \pm 0.28^{* *}$ & $9.38 \pm 0.60^{*}$ & $8.39 \pm 1.11^{*}$ & $9.79 \pm 0.94$ \\
72 & $9.80 \pm 0.60$ & $10.11 \pm 0.22$ & $8.53 \pm 0.88^{*}$ & $9.11 \pm 0.85^{*}$ \\
7 day & $10.16 \pm 0.56$ & $9.81 \pm 0.22^{*}$ & $8.98 \pm 0.52^{* *}$ & $9.01 \pm 0.48^{*}$ \\
\hline
\end{tabular}

a, Number of rats; b, Mean \pm S.E.; *, $\mathbf{P}<0.05 ; * *, \mathbf{P}<0.01$ vs. the value at 0 hr in each group. 
showed a further decrease at $48 \mathrm{hr}$ (Fig. 1). Ten weeks later the calcium level in male rats in this group was still as low as 8.31 $\pm 0.56 \mathrm{mg}$ calcium $/ 100 \mathrm{ml}$ serum and the level in female rats was higher than that in males (Fig. 1). No significant fall in calcium level but a tendency to increase toward the normal level was shown in both male and female rats following bilateral thyroidectomy (Fig. 1). The magnitude in the increase in calcium level was much greater in females than that in males; The calcium level returned to the normal range in female rats.

One week after the first unilateral parathyroidectomy, the serum calcium level in male and female rats was in a subnormal range (Fig. 2). When the second unilateral parathyroidectomy was performed in these rats, the serum calcium level in male rats did not show any significant fall during the $48 \mathrm{hr}$ observation and the level in female rats showed a slight decrease at $10 \mathrm{hr}$ and an increase to a nearly normal level at 24 $\mathrm{hr}$. The serum calcium level in both sexes was in the normal range when measured 10 weeks later (Fig. 2). In these male rats, thyroidectomy produced a marked fall in the serum calcium level within $2 \mathrm{hr}$ and a progressive return to the initial level by the 7th day. In female rats, no decrease but a gradual increase in serum calcium over the normal level was noted following thyroidectomy (Fig. 2).

The initial calcium level in female rats was slightly higher than that in normal rats when the second unilateral parathyroidectomy was performed 6 weeks after the last operation, and the level showed a significant decrease after the second unilateral parathyroidectomy but it was still maintained in the normal or subnormal range until the 7 th day after the second operation (Fig. 3). The calcium level in male rats was also normal just before the second unilateral parathyroidectomy and a less marked decrease was shown until $24 \mathrm{hr}$. However, the calcium level in these male rats showed a gradual decrease during the

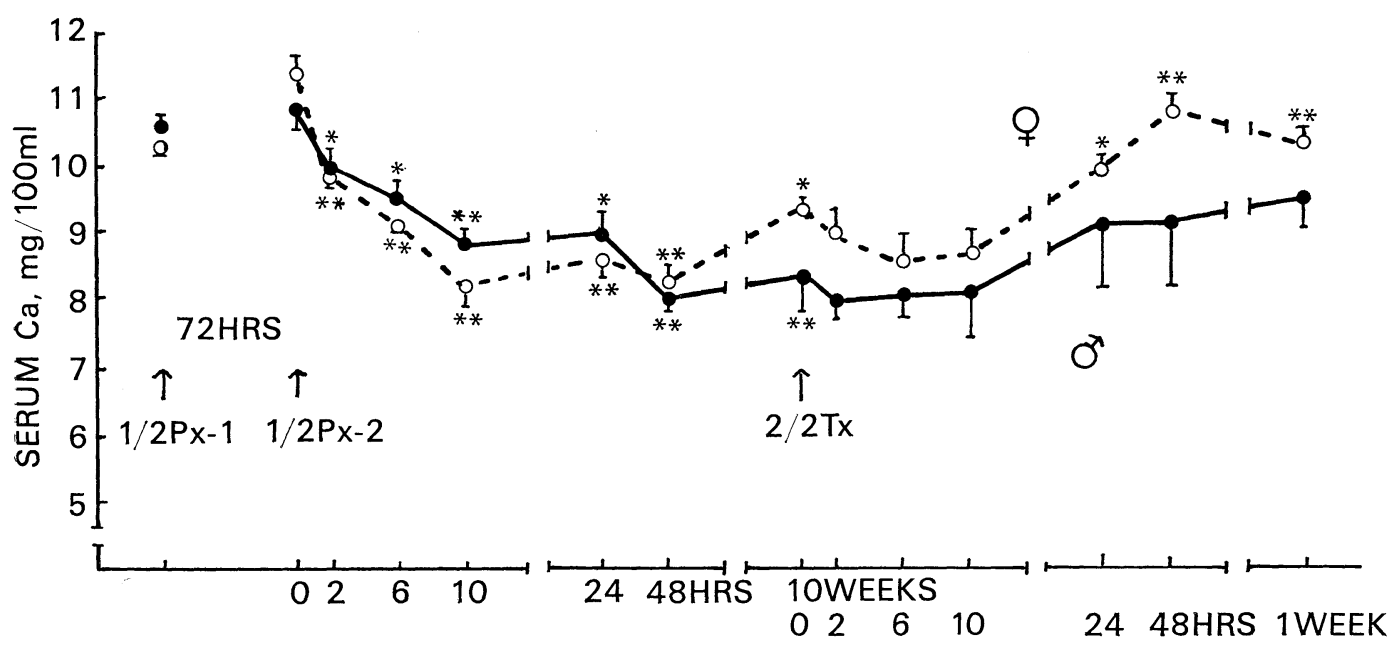

TIME AFTER OPERATION

Fig. 1. Time course of the changes in serum calcium level in adult male and female rats parathyroidectomized unilaterally $(1 / 2 \mathrm{Px}-2) 72 \mathrm{hr}$ after the first unilateral parathyroidectomy $(1 / 2 \mathrm{Px}-1)$ and thyroidectomized bilaterally $(2 / 2 \mathrm{Tx}) 10$ weeks after the second unilateral parathyroidectomy. Vertical lines represent the standard error of the mean of 6 animals.

$*, \mathrm{P}<0.05 ; * *, \mathrm{P}<0.01$ vs. the value at $0 \mathrm{hr}$ of each operation. 


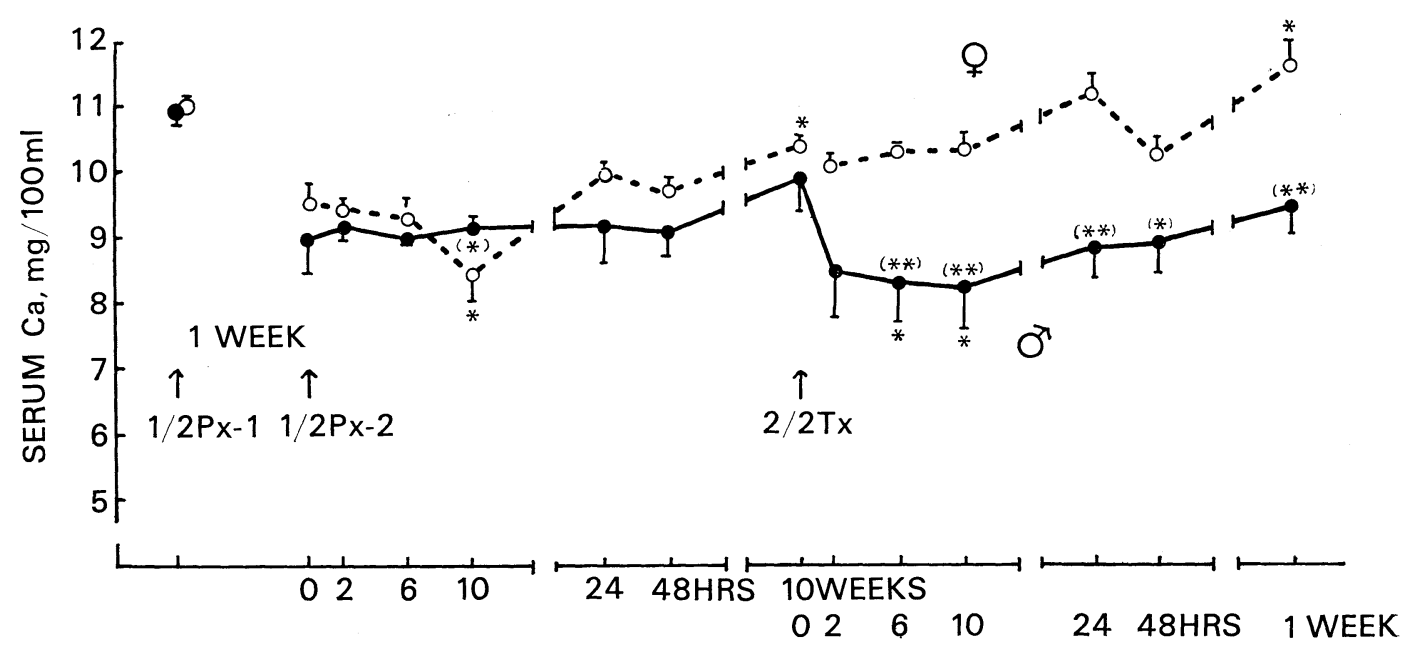

TIME AFTER OPERATION

Fig, 2. Time course of the changes in serum calcium level in adult male and female rats parathyroidectomized unilaterally $(1 / 2 \mathrm{Px}-2)$ one week after the first unilateral parathyroidectomy $(1 / 2 \mathrm{Px}-1)$ and thyroidectomized $(2 / 2 \mathrm{Tx})$ bilaterally 10 weeks after the second unilateral parathyroidectomy. Vertical lines represent the standard error of the mean of 6 animals.

$*, \mathrm{P}<0.05 ; * *, \mathrm{P}<0.01$ vs. the value at $0 \mathrm{hr}$ of each operation. $(*), \mathrm{P}<0.05 ; \quad(* *,, \mathrm{P}<0.01$ vs. the value of females at the corresponding hr.

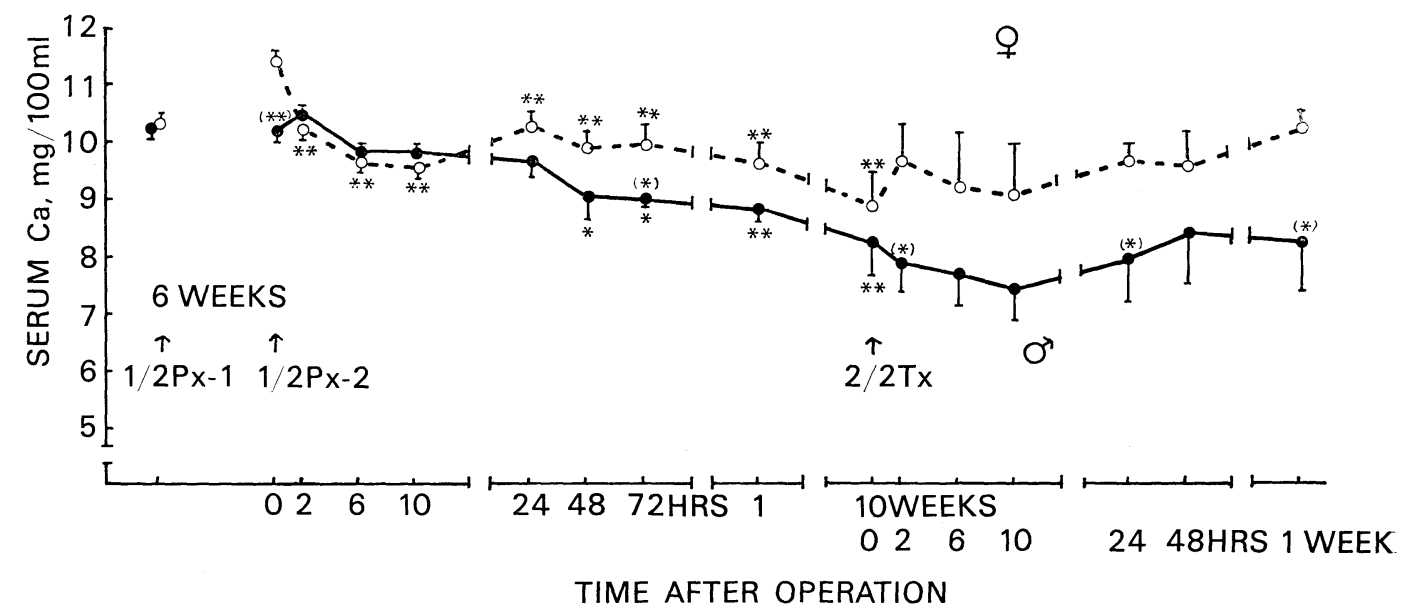

Fig. 3. Time course of the changes in serum calcium level in adult male and female rats parathyroidectomized unilaterally $(1 / 2 \mathrm{Px}-2) 6$ weeks after the first unilateral parathyroidectomy $(1 / 2 \mathrm{Px}-1)$ and thyroidectomized bilaterally $(2 / 2 \mathrm{Tx}) 10$ weeks after the second unilateral parathyroidectomy. Vertical lines represent the standard error of the mean of 5 animals.

$*, \mathrm{P}<0.05 ; * *, \mathrm{P}<0.01$ vs. the value at $0 \mathrm{hr}$ of each operation. $(*), \mathrm{P}<0.05$ vs. the value of females at the corresponding hr. 


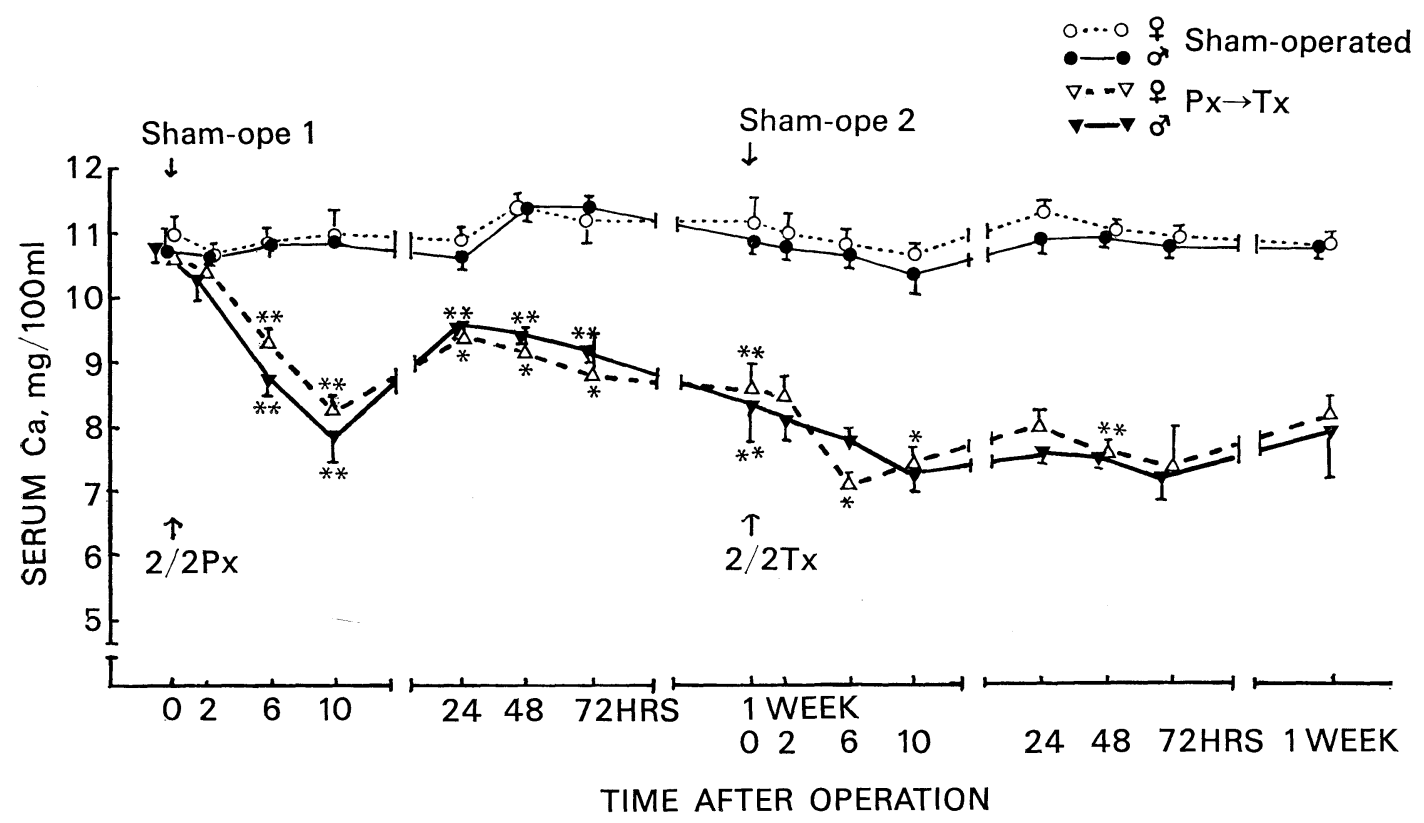

Fig. 4. Time course of the changes in serum calcium level in adult male and female rats parathyroidectomized bilaterally $(2 / 2 \mathrm{Px})$ and thyroidectomized bilaterally $(2 / 2 \mathrm{Tx})$ one week later. Control animals were sham-operated twice as indicated by an arrow. Vertical lines represent the standard error of the mean derived from 5 rats.

*, $\mathrm{P}<0.05 ; * *, \mathrm{P}<0.01$ vs. the value at $0 \mathrm{hr}$ of each operation.

subsequent one week period. At 10 weeks post-operation serum calcium concentrations in both male and female rats were significantly low. In male rats, a slight and transient decrease in serum calcium was evident until $10 \mathrm{hr}$ after thyroidectomy and the level did not increase beyond the initial level. On the other hand, no further decrease was shown in female rats following thyroidectomy and a gradual increase toward the normal level was seen during one week post-operation.

Effect of simultaneous removal of bilateral parathyroid glands and the following thyroidectomy

Simultaneous removal of bilateral parathyroid glands led to a prompt fall in the serum calcium level in $6 \mathrm{hr}$ following the operation (Fig. 4). The calcium level reached the lowest level at $10 \mathrm{hr}$ and tended to increase toward the control level at $24 \mathrm{hr}$ but the significantly low level was maintained for the period of one week-observation. The decrease in calcium level in male rats was slightly greater than that in females.

Removal of thyroid glands in these rats, one week later, produced a further decline in serum calcium and no significant sex difference in the changing pattern of calcium level was shown (Fig. 4). Serum calcium in sham-operated rats fluctuated slightly throughout the experimental period (Fig. 4). Simultaneou removal of bilateral thyroid glands demonstrated a very similar pattern of the time course changes in serum calcium as that seen after simultaneous bilateral parathyroidectomy (Table 1). Time course changes in the serum protein concentrations was studied following bilateral thyroparathyroidectomy and thyroidectomy. 
Table 2. Time course changes in serum protein concentrations $(\mathrm{mg} / 100 \mathrm{~m} l)$ following bilateral surgical parathyroidectomy and thyroidectomy in adult male and female rats. Thyroidectomy was performed one week after parathyroidectomy. The animals were fed ad libitum throughout the experiment.

\begin{tabular}{|c|c|c|c|c|c|c|}
\hline \multirow{2}{*}{$\begin{array}{l}\text { Time after } \\
\text { operation }\end{array}$} & \multicolumn{3}{|c|}{ Parathyroidectomy } & \multicolumn{3}{|c|}{ Thyroidectomy } \\
\hline & Male $(5)^{\mathrm{a}}$ & & Female (5) & Male (5) & & Female (5) \\
\hline $0 \mathrm{hr}$ & $7.5 \pm 0.3^{b}$ & $\mathbf{N} \mathbf{S}$ & $7.8 \pm 0.2$ & $7.5 \pm 0.2$ & N S & $7.3 \pm 0.3$ \\
\hline 2 & $7.0 \pm 0.3$ & (N.S.) & $7.0 \pm 0.1$ & $6.5 \pm 0.2^{* *}$ & (N.S.) & $6.6 \pm 0.3$ \\
\hline 6 & $6.9 \pm 0.2$ & (N.S.) & $7.6 \pm 0.2$ & $6.8 \pm 0.2^{*}$ & (N.S.) & $6.6 \pm 0.3$ \\
\hline 10 & $6.7 \pm 0.3$ & (N.S.) & $7.0 \pm 0.2$ & $6.6 \pm 0.2^{*}$ & (N.S.) & $6.8 \pm 0.1$ \\
\hline 24 & $7.2 \pm 0.4$ & (N.S.) & $7.4 \pm 0.3$ & $7.4 \pm 0.1$ & (N.S.) & $6.9 \pm 0.2$ \\
\hline 48 & $7.5 \pm 0.4$ & (N.S.) & $6.9 \pm 0.3^{*}$ & $7.5 \pm 0.3$ & (N.S.) & $7.5 \pm 0.2$ \\
\hline 72 & $7.4 \pm 0.3$ & (N.S.) & $6.7 \pm 0.4$ & $7.4 \pm 0.4$ & (N.S.) & $6.6 \pm 0.3$ \\
\hline 7 day & $7.5 \pm 0.2$ & (N.S.) & $7.3 \pm 0.3$ & $6.4 \pm 0.2^{*}$ & (N.S.) & $6.6 \pm 0.3$ \\
\hline
\end{tabular}

a, Number of rats; b, Mean \pm S.E.; $*, \mathrm{P}<0.05 ; * *, \mathrm{P}<0.01$ vs. the value at $0 \mathrm{hr} ; \mathrm{N} . \mathrm{S}$, not significantly different between sexes.

No significant fluctuation in the level of serum protein was observed (Table 2).

\section{Discussion}

The present results clearly show that adaptation of the regulatory system of serum calcium to the absence of parathyroid hormone occurrs in adult rats if parathyroid hormone is deprived gradually, and that particularly female rats possess a greater ability to restore the serum calcium level toward normal range without parathyroid hormone. In addition, it is obviously shown that adaptation to unilateral parathyroidectomy requires a certain period of time, at least more than $72 \mathrm{hr}$ during which the regulatory system of serum calcium becomes able to maintain the calcium level nearly normal when the parathyroid hormone is completely deprived thereafter.

Removal of parathyroid gland has been well known to manifest a low serum calcium level. The concept of the calcium homeostasis is that parathyroid hormone mobilizes bone calcium and suppresses the secretion of calcium into the urine (for Review, see Copp, 1969). Intestinal calcium absorption appears to be regulated by 1, 25-dihydroxyvitamin $D_{3}$ (Norman and Henry, 1974). Recently, it has been considered that blood calcium level is set in the normal animal largely by the rates of calcium absorption in the intestine and reabsorption in the kidney in addition to the bone resorption (Kalu et al., 1974; Reynolds, 1975).

A prompt fall in the serum calcium level seen after bilateral parathyroidectomy in fasting mature rats has been explained as to be due to calcitonin dominant state resulted from the loss of parathyroid hormone (Kalu et al., 1976). In the present experiment, activity of calcitonin producing cells could be reduced during the adaptation to the first unilateral parathyroidectomy because physiological role of calcitonin has been considered to suppress the calcium level if increased over the normal range, by mediation of suppressing bone resorption (Munson and Gray, 1970). Therefore, if serum calcium level is reduced by unilateral 
parathyroidectomy the production and release of calcitionin might be decreased by mediation of feedback control mechanism dependent upon the period of exposure time to a lower level of calcium. In addition, the rest of the parathyroid gland may become to compensate for the removed parathyroid gland in one to six weeks. These facts might lead to alterations in the response of the kidney, intestine and bone to restore the serum calcium, and this could reflect in the less marked decrease in the serum calcium in the acute phase following the second unilateral parathyroidectomy. However, sex difference in adaptive changes in serum calcium level shown after the second unilateral parathyroidectomy can not be explained by the reduced level of calcitonin. It has been reported that men has higher basal plasma concentrations of immunoreactive calcitonin than women and calcitonin release in response to hypercalcemia in men is larger than in women (Heath and Sizemore, 1977), However, restoration in serum calcium after removal of thyroid gland with thyrocalcitonin producing cells was greater in females than in males in the present experiment.

It is interesting to note that this sex difference appeared only when thyroidectomy was carried out after parathyroid hormone was gradually removed. Thyroidectomy performed one week after simultaneous bilateral parathyroidectomy resulted in a further decline in the serum calcium level to a similar extent in both male and female animals. There are several reports on the implication of estrogens in the calcium metabolism. Estrogens antagonize the action of calcitonin resulting in the disappearance of lowering effect of serum calcium by calcitonin (Shai and Wallach, 1973). Noble and Matty (1967) have suggested that thyroid hormone could directly inhibit the intestinal calcium absorption. The fact that the maintenance of subnormal or normal calcium level in female rats seen even after bilateral parathyroidectomy would suggest the implication of estrogens antagonizing with calcitonin, but the increase in the serum calcium level after thyroidectomy is not attributable to antagonism by estrogens with calcitonin because of the absence of calcitonin. Release from the inhibiting action of thyroid hormone in calcium absorption is also inadequate to explain the sex difference in the adaptive changes in serum calcium following bilateral thyroidectomy. Stimulatory effect of estrogens on the production of 1,25-dihydroxyvitamin $\mathrm{D}_{3}$ in the kidney (Tanaka, et al., 1976; Baksi and Kenny, 1977), though this effect was mainly observed in birds, which results in increasing the calcium absorption from the intestine could be a major factor to maintain the serum calcium level under the conditions where parathyroid hormone, calcitonin and thyroid hormones were absent.

Various factors other than the three major hormones affecting calcium metabolism have been reported. Prostaglandin $\mathrm{E}_{2}$ which produces an elevation of the serum calcium in intact rats when infused continuously (Franklin and Tashjian, 1975), the osteoclast-activating factor of lymphoid cells in elevating serum calcium level via bone resorption (Horton et al., 1972) or any unknown factors can be involved to control the level of serum calcium under the conditions where parathyroid hormone is deprived, and/or where thyroid hormone and calcitonin are deprived. The rise in serum calcium has been noted in chronically parathyroidectomized rats adapted to the higher calcium diet (Kemm, 1976). No significant rise in serum calcium level in chronically parathyroidectomized rat has been shown if they were fed the low calcium diet as likely as $0.2 \%$ calcium. In this situation, possible role of 1,25-dihydroxycholecalciferol has not been proved by $\mathrm{Kemm}$ (1976). Concentration of serum proteins is well known to affect the serum calcium level. There was no significant 
sex difference in serum protein concentration during one week following bilateral parathyroidectomy or bilateral thyroidectomy, though the serum protein concentration in male rats decreased significantly until $10 \mathrm{hr}$ after thyroidectomy. Therefore, sex difference in the calcium levels in the present experiment could not be the result from the sex difference in protein metabolism. Sammon et al. (1970) and Fleisch et al. (1975) have also demonstrated that rats are able to adapt calcium release from bone without parathyroid hormone or calcitonin, though the mechanism is unknown. Detailed mechanisms of adaptation to the gradual deprivation of parathyroid hormone must be clarified in relation to the response of intestine, kidney and bone in further experiments.

\section{Acknowledgements}

We are grateful to Miss N. Yamamoto for her $^{2}$ technical assistance.

\section{References}

Baksi, S. N. and A. D. Kenny (1977). Endocrinology 101, 1216.

Copp, D. H. (1969). Ann. Rev. Pharmacol. 9, 327.

Fleisch, H., B. Haldimann and J.-P. Bonjour (1975). Int. Congr. Series. 346, 262.

Franklin, R. B. and A. H. Tashjian, Jr. (1975). Endocrinology 97, 240.

Fujii, T. (1978). Nature 273, 236.

Heath, H. III. and G. W. Sizemore (1977). J. Clin. Invest. 60, 1135.

Horton, J. E., L. G. Raisz, H. A. Simmons and J. J. Oppenheim (1972). Science 177, 793.

Kalu, D. N., A. Hadji-Georgopoulos, M. G. Sarr, B. A. Solomon and G. V. Foster (1974). Endocrinology 95, 1156.

Kalu, D. N., A Hadji-Georgopoulos and G. V. Foster (1976). Endocrinology 98, 534.

Kemm, J. R. (1976). J. Physiol. 256, 103.

Lowry, O. H., N. J. Rosebrough, A. L. Farr and R. J. Randall (1951). J. Biol. Chem. 193, 265.

Munson, P. L. and T. K. Gray (1970). Fed. Proc. 29,1206 .

Noble, H. M. and A. J. Matty (1967). J. Endocrinol. $37,111$.

Norman, A. W. and H. Henry (1974). Recent. Prog. Horm. Res. 30, 431.

Reynolds, J. J. (1975). Int. Congr. Series. 346, 254.

Sammon, P. J., R. E. Stacey and F. Bronner (1970). Amer. J. Physiol. 218, 479.

Shai, F. and S. Wallach (1973). Endocrinology 93, 1044.

Tanaka, Y., L. Castillo and H. F. DeLuca (1976). Proc. Natl. Acad. Sci. USA. 73, 2701. 